\title{
Isolation and characterization of a thermophilic sulfur- and iron- reducing thaumarchaeote from a terrestrial acidic hot spring
}

\author{
Shingo Kato $\mathbb{D}^{1,2} \cdot$ Takashi Itoh $^{1} \cdot$ Masahiro Yuki $^{1} \cdot$ Mai Nagamori, ${ }^{1,3}$ Masafumi Ohnishi, ${ }^{1,3}$ Katsuyuki Uematsu $^{4} \cdot$ \\ Katsuhiko Suzuki $\mathbb{D}^{2} \cdot$ Tomonori Takashina $^{3} \cdot$ Moriya Ohkuma $^{1}$
}

Received: 10 September 2018 / Revised: 18 April 2019 / Accepted: 3 May 2019 / Published online: 6 June 2019

(c) International Society for Microbial Ecology 2019

\begin{abstract}
A deep-branching clade of Thaumarchaeota, conventionally called Terrestrial hot spring creanarchaeotic group (THSCG), is a missing link between thaumarchaeotic ammonia oxidizers and the deeper-branching non-ammonia oxidizers, such as Crenarchaeota and Candidatus Korarchaeota. Here, we report isolation of the first cultivated representative from the THSCG, named as NAS-02. Physiological characterization demonstrated that the isolate was a thermoacidophilic, sulfurand iron-reducing organoheterotroph, which was supported by gene contents encoded in its complete genome. There was no evidence for ammonia oxidation by the isolate. Members in THSCG are likely thermophiles, and may play roles in degrading cell debris as a scavenger and in biogeochemical cycling of sulfur and iron in the hot environments, as suggested by the physiological characteristics of the isolate and the geographical distribution of the 16S rRNA gene sequences of THSCG in terrestrial hot springs and marine hydrothermal fields. Phylogenetic analysis suggests that the THSCG lineage represented by NAS-02 has gained the ability of sulfur reduction via horizontal gene transfer. Based on the phylogeny and physiology, we propose the name Conexivisphaera calidus gen. nov., sp. nov. to accommodate the isolate.
\end{abstract}

\section{Introduction}

Archaea are ubiquitous and play a significant role in biogeochemical cycling in the environment. Recent cultureindependent metagenomics and single-cell genomics have allowed to determine genome sequences of individuals of

Supplementary information The online version of this article (https:// doi.org/10.1038/s41396-019-0447-3) contains supplementary material, which is available to authorized users.

Shingo Kato

skato@ riken.jp

1 Japan Collection of Microorganisms, RIKEN BioResource Research Center, 3-1-1 Koyadai, Tsukuba, Ibaraki 305-0074, Japan

2 Ore Genesis Research Unit, Project Team for Development of New-generation Research Protocol for Submarine Resources, Japan Agency for Marine-Earth Science and Technology (JAMSTEC), Yokosuka, Kanagawa 237-0061, Japan

3 Graduate School of Life Sciences, Toyo University, Oura, Gunma 374-0193, Japan

4 Department of Marine and Earth Sciences, Marine Work Japan Ltd, Yokosuka, Kanagawa 237-0061, Japan novel archaeal clades without isolated representatives [1-6]. These findings have strongly pushed forward our knowledge of the phylogenetic diversity and metabolic potentials in the Archaea, and moreover, evolution of life. Remarkably, all archaeal isolates reported so far belong to only several clades in Crenarchaeota, Euryarchaeota, and Thaumarchaeota. The physiology of the remaining uncultured archaeal clades has not been demonstrated yet [7,8].

Terrestrial hot springs harbor diverse as-yet-uncultured archaea in higher-taxonomic-level clades [9-11]. One of the uncultured clades is the Terrestrial hot spring creanarchaeotic group (THSCG) [12], also referred to as Hot Thaumarchaetota-related Clade (HTC) [13]. THSCG is phylogenetically located between ammonia-oxidizing thaumarchaeota (AOT) and deeper lineages, such as $\mathrm{Cre}$ narchaeota, Ca. Aigarchaeota, and Ca. Korarchaeota [14]. Thus, THSCG is a key group to understand the evolution of Archaea from non-ammonia-oxidizers to AOT that play a significant role in biogeochemical cycling of nitrogen and carbon in the present Earth [15-17]. Metagenomeassembled genomes (named Beowulf BS1 and Dragon DS1) reported in hot springs in Yellowstone National Park, USA, have suggested that THSCG contains sulfur-oxidizing nitrate-/oxygen-reducers and heterotrophic sulfur-reducers 
[18]. However, the ecophysiology of THSCG is still poorly understood because of the absence of any isolated representative. Here, we report the isolation and characterization of a sulfur- and iron-reducing thermoacidophilic archaeon, strain NAS-02, belonging to THSCG. We performed physiological, morphological and genomic analyses of this first isolate of THSCG.

\section{Materials and methods}

\section{Sampling and site description}

We collected fluid samples at a thermoacidic spring at OkuShiobara, Tochigi, Japan (36 $57^{\prime} 17^{\prime \prime}$ N, 139 $46^{\prime} 47^{\prime \prime}$ E), as previously described [19]. The fluid chemistry of the upper, middle and lower points of a stream (Fig. S1; Table S1) were measured in April 2012. Temperature, $\mathrm{pH}$ and dissolved $\mathrm{O}_{2}$ concentration were measured using a portable meter (D-55; Horiba, Kyoto, Japan). Salinity was measured using a hand-held refractometer (MASTER-S/Millo; Atago, Tokyo, Japan). Concentration of total sulfide ion was measured using a Gastec detector tube (Type 211; Kanagawa, Japan). Concentration of $\mathrm{Fe}^{2+}$ was measured by ferrozine assay [20]. For PCR clone analysis of 16S rRNA genes, we collected the fluid samples $(1 \mathrm{~L}$ for each sampling point). The collected samples were stored with cold storage packs in a cooler box, brought back to our laboratory, and filtered using $0.2-\mu \mathrm{m}$-pore-sized membrane filters within a day. The filters were stored in $-20{ }^{\circ} \mathrm{C}$ until DNA extraction. For cultivation, fluid samples were collected into sterile glass bottles at the middle point of the stream in August 2012. The collected samples were brought back to our laboratory without cooling, inoculated into an enrichment medium, and incubated within a day.

\section{Enrichment and isolation}

Using the collected sample from the middle stream as an inoculum, we performed enrichment cultivations with the culture medium contained (per liter); $0.2 \mathrm{~g}\left(\mathrm{NH}_{4}\right)_{2} \mathrm{SO}_{4}, 3.0 \mathrm{~g}$ $\mathrm{KH}_{2} \mathrm{PO}_{4}, 0.5 \mathrm{~g} \mathrm{MgSO}_{4} .7 \mathrm{H}_{2} \mathrm{O}, 0.25 \mathrm{~g} \mathrm{CaCl}_{2} .2 \mathrm{H}_{2} \mathrm{O}, 1.8 \mathrm{mg}$ $\mathrm{MnCl}_{2} .4 \mathrm{H}_{2} \mathrm{O}, 4.5 \mathrm{mg} \mathrm{Na}_{2} \mathrm{~B}_{4} \mathrm{O}_{7} .10 \mathrm{H}_{2} \mathrm{O}, 0.22 \mathrm{mg} \mathrm{ZnSO}{ }_{4} .7 \mathrm{H}_{2} \mathrm{O}$, $0.05 \mathrm{mg} \quad \mathrm{CuCl}_{2} .2 \mathrm{H}_{2} \mathrm{O}, 0.03 \mathrm{mg} \quad \mathrm{Na}_{2} \mathrm{MoO}_{4} .2 \mathrm{H}_{2} \mathrm{O}, 0.03 \mathrm{mg}$ VOSO $_{4} \cdot \mathrm{nH}_{2} \mathrm{O}, 0.01 \mathrm{mg} \mathrm{CoSO}_{4} \cdot 7 \mathrm{H}_{2} \mathrm{O}, 0.5 \mathrm{~g}$ yeast extract (BD-Difco), $10 \mathrm{mM} \mathrm{Fe(III)-citrate} \mathrm{and} 10 \mathrm{ml}$ vitamin mixture [21]; $\mathrm{pH}$ adjusted to 5.0. The medium was placed in culture bottles under a gas phase of $\mathrm{N}_{2} / \mathrm{CO}_{2}(4: 1, \mathrm{v} / \mathrm{v})$, and reduced with $0.25 \mathrm{mM}$ L-cysteine. $\mathrm{HCl}$ and $1.3 \mathrm{mM}$ $\mathrm{FeCl}_{2} \cdot 4 \mathrm{H}_{2} \mathrm{O}$. The cultivation was performed at $70^{\circ} \mathrm{C}$ for 1 month, and microbial growth was observed in the enrichment culture by phase-contrast microscopy. An archaeal strain was isolated by a repeated dilution-to- extinction method, designated as NAS-02. Purity of the strain was confirmed by morphological observation and the 16S rRNA gene clone analysis as previously reported [19] using bacterial and archaeal universal primers: Arch9F [10] and Uni1406R [22] for archaea, and EB-20F [23] and Uni1406R for bacteria. The purity was also proved by the whole-genome sequencing, showing that only the sequences of the NAS-02 genome were detected.

\section{Physiological characterization}

During growth characterization, NAS-02 was found to grow better in a modified enrichment medium supplemented with $2.5 \mathrm{~g} \mathrm{~L}^{-1} \mathrm{Na}_{2} \mathrm{~S}_{2} \mathrm{O}_{3} .5 \mathrm{H}_{2} \mathrm{O}$, instead of $\mathrm{Fe}$ (III)-citrate. Unless otherwise indicated, it was routinely cultivated with $\mathrm{S}_{2} \mathrm{O}_{3}{ }^{2-}$ at $65{ }^{\circ} \mathrm{C}$ at $\mathrm{pH} 5.0$ under $\mathrm{N}_{2}-\mathrm{CO}_{2}(4: 1, \mathrm{v} / \mathrm{v})$. NAS-02 was tested for growth on organic and inorganic substrates. As electron acceptors, $\mathrm{S}_{2} \mathrm{O}_{3}{ }^{2-}, \mathrm{SO}_{4}{ }^{2-}, \mathrm{NO}_{3}{ }^{-}, \mathrm{S}^{0}$, and $\mathrm{Fe}$ (III) citrate were added (final $20 \mathrm{mM}$ ). For the gas phase composition, $\mathrm{N}_{2}, \mathrm{~N}_{2}-\mathrm{CO}_{2}\left(80: 20\right.$, v/v), $\mathrm{H}_{2}-\mathrm{CO}_{2}\left(80: 20\right.$, v/v), $\mathrm{N}_{2}-$ $\mathrm{CO}_{2}-\mathrm{O}_{2}(79: 20: 1, \mathrm{v} / \mathrm{v} / \mathrm{v})$, and $\mathrm{N}_{2}-\mathrm{CO}_{2}-\mathrm{O}_{2}(78: 20: 2, \mathrm{v} / \mathrm{v} / \mathrm{v})$ were tested. Growth at various $\mathrm{pHs}$ was examined with the following buffers (10 mM each): $\mathrm{pH}$ 4.0-5.5, HOMOPIPES: pH 5.0-7.0, MES: 6.5-8.0, HEPES. The $\mathrm{pH}$ of the culture medium was adjusted from 3.5 to 6.5 at 0.5 intervals. The cultivations were performed at $55^{\circ} \mathrm{C}$ to $80^{\circ} \mathrm{C}$ at $5{ }^{\circ} \mathrm{C}$ intervals. As electron donors and carbon sources, a variety of organic carbon compounds, such as sugars, organic acids, alcohols and proteinaceous substances, were added (Table S2). Growth was determined using a florescence microscopy (BX51, Olympus, Japan) by direct counts of cells stained with SYBR green I.

\section{Morphology}

Morphology of cells was observed by phase-contrast microscopy and transmission electron microscopy (TEM). For preparation of ultra-thin sections for TEM, we employed a method using freeze-substitution after glutaraldehyde fixation [24] with minor modifications to preserve ultrastructure of the cells. The cells grown at $70^{\circ} \mathrm{C}$ for 3 weeks were fixed with formaldehyde (final 4\%) in the culture at $70{ }^{\circ} \mathrm{C}$ for $30 \mathrm{~min}$, and then these were fixed with glutaraldehyde (final 2.5\%) in the culture at room temperature. The fixed specimens were frozen in a highpressure freezing apparatus (EM-PACT2; Leica, Wetzlar, Germany). The frozen samples were substituted with $\mathrm{OsO}_{4}$ (final $2 \%$ ) in acetone for 4 days at $-80{ }^{\circ} \mathrm{C}$. The samples were warmed gradually to room temperature, rinsed with acetone, stained with uranyl acetate (final 2\%) in methanol, and then embedded in epoxy resin (TAAB Laboratories Equipment Ltd, Aldermaston, UK). Ultra-thin sections (70 $\mathrm{nm}$ in thickness) were prepared using an ultra- 
microtome (EM-UC7; Leica), and were stained with uranyl acetate (final 2\%) and lead-stained solution (lead nitrate and lead acetate, final $0.3 \%$ each). The sections were observed by a TEM (Tecnai G2 20; FEI, Hillsboro, OR, USA) at an acceleration voltage of $120 \mathrm{kV}$. We performed elemental analysis using energy dispersive X-ray spectrometry (EDS) (EDAX EDS System; AMETEK, Inc., Minneapolis, MN, USA) equipped with the TEM.

\section{Genome analysis}

Genomic DNA was extracted from cell suspension of NAS-02 after 40 days cultivation as previously described [19]. The DNA library was constructed using a Nextera XT DNA Library Prep Kit (Illumina, San Diego, CA, USA), and genome sequencing was performed on the Illumina MiSeq platform with MiSeq Reagent Kit v3 (2x300). The extracted DNA was also applied to wholegenome sequencing using an Ion Torrent PGM system (Life Technologies) and 454 pyrosequencing (Roche) at the Center for Omics and Bioinformatics of the University of Tokyo, Japan. We performed removal of adapter sequences from reads, removal of the reads shorter than $100 \mathrm{bps}$, and trimming of low-quality ends of the reads using CLC Genomics Workbench version 9.5.3 (QIAGEN Aarhus A/S) with the default setting. All the treated reads were assembled using SPAdes [25] version 3.9.0 with the following parameter $(-\mathrm{k} 33,55,77,99)$, resulting in six contigs. Gaps were closed by sanger sequencing of PCRamplified gap regions. The genome sequences were annotated using Prokka [26] version 1.11 that includes Prodigal [27], Infernal [28] and RNAmmer [29] for detection of protein-coding DNA sequences (CDSs), noncoding RNA and rRNA, respectively. The tRNAs in the genome were predicted using tRNAscan-SE v.2.0 [30]. We also used RAST [31] version 2.0 for gene prediction and annotation. The results of gene prediction and annotation were manually checked and curated. The position of OriC was predicted based on the previous report [32]. Homology searching of all of the CDSs against a nonredundant (nr) database in NCBI (downloaded at 12 April 2018) were performed using Blastp [33] version 2.7.1 with the default parameters. Using the searching result with top 100 hits, the taxonomic classification of the CDSs were determined using MEGAN community edition [34] version 6.11 .2 with the default setting by the lowest common ancestor algorithm [35]. All of the CDSs were classified into Orthologous Groups (OGs) using eggNOG version 4.5.1 [36]. The prediction of metabolic pathways was performed using the Kyoto Encyclopedia of Genes and Genomes (KEGG) pathway tool [37]. Functional properties of proteins coded by CDSs were predicted using InterProScan [38] version 5.24-63.0. Extracellular transport signals of CDSs were identified using PSORTb 3.0 [39]. The peptidase database MEROPS was used to classify peptidase-like CDSs [40]. Optimal growth temperatures were estimated from genome sequences using Tome version 1.1 [41].

\section{Phylogenetic analysis}

To construct a phylogenetic tree for the 16S rRNA gene of NAS-02, its relatives were collected from public databases, such as Genbank/DDBJ/EMBL and Silva database [42]. The sequences of NAS-02 and the relatives were aligned by SINA [43] using an aligned sequences curated by Silva [42] as a reference. The alignment was trimmed using TrimAl [44] version 1.2re59 with the "-automated1" option. The trimmed alignment was used for Maximum-Likelihood tree construction using RAxML [45] version 8.2.10 with the GTRGAMMA model. To construct a genome tree, i.e., a phylogenetic tree of concatenated amino acid sequences from the 43 single copy marker proteins (Table S3) as determined previously [46], the dataset of the sequences were collected from the databases and aligned using MUSCLE [47] version 3.8.31. Based on the alignment trimmed using TrimAl, the genome tree was constructed using RAxML with the PROTOGAMMALG model. We also constructed phylogenetic trees of amino acid sequences encoded by each CDS as described above. For all trees, bootstrap support values were computed with 1000 resamples.

\section{S rRNA gene survey}

The 16S rRNA genes related to THSCG deposited into Sequence Read Archive (SRA) in National Center for Biotechnology Information (NCBI), which were produced by next-generation sequencing methods, such as pyrosequencing and Illumina technology, were surveyed using an IMNGS (Integrated Microbial Next-Generation Sequencing) platform [48] with a 95\% similarity threshold. In this survey, we used 10 representative sequences of the reported THSCG 16S rRNA genes (AB007307, DQ834113, AB007308, EF156533, HE574567, AB019732, DQ243777, and FJ797319 for NCBI accession number, 2263083025 (DS1) for JGI-IMG accession number, and that of NAS02) as queries. The 16S rRNA genes affiliated in THSCG in the Silva database release 132 were also surveyed.

\section{S rRNA gene PCR clone analysis}

Genomic DNA was extracted from the membrane filters as described above. Archaeal 16S rRNA genes were amplified by PCR using Arc9F and Uni1406R, and the PCR products were cloned and sequenced as previously described [10]. 


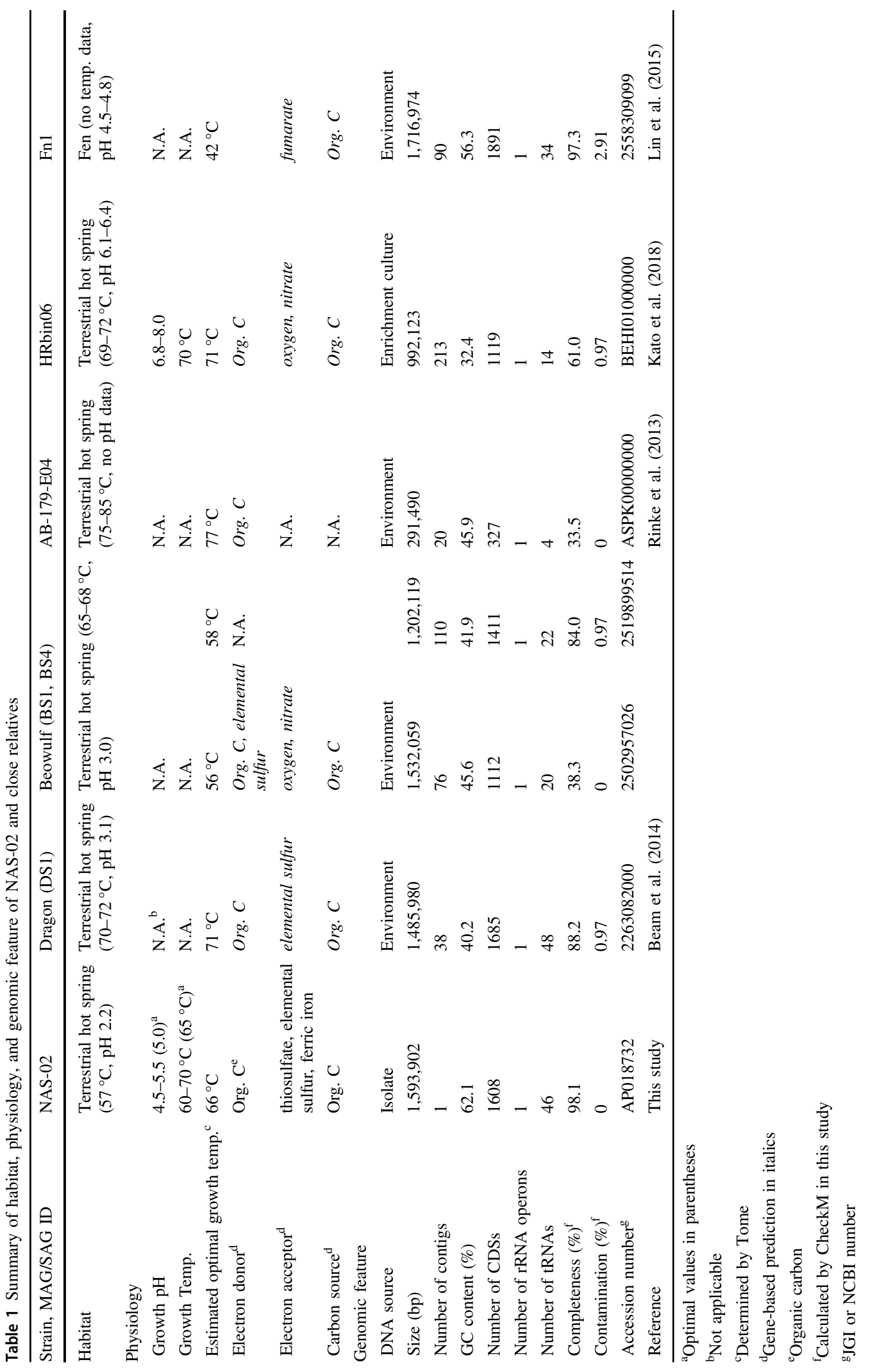


QIIME 1.9.1 [49] was used for chimera removal, OTU clustering, and taxonomic affiliation of the obtained sequences. The detected clones and close relatives were collected from public databases, the sequences were aligned using SINA, gap positions were removed, and a ML tree of the $16 \mathrm{~S}$ rRNA sequences was constructed using RAxML, as described above.

\section{Accession number}

The complete genome sequence of the NAS-02 was deposited into the DDBJ under the following accession number, AP018732. The archaeal 16S rRNA genes used in the clone library analysis were deposited into the DDBJ under the following accession numbers; AB931042-AB931105, LC158371-LC158446, and AB930951-AB931041, for the upper, middle, and lower stream samples, respectively.

\section{Results and discussion}

We isolated NAS-02 from a thermoacidic water sample $\left(57^{\circ} \mathrm{C}\right.$ and $\left.\mathrm{pH} 2.2\right)$ using an anaerobic medium amended with $\mathrm{Fe}(\mathrm{III})$-citrate as the sole electron acceptor. We determined its complete genome sequence (Table 1, Fig. S2, Table S4), and constructed phylogenetic trees with a concatenated alignment of conserved single copy marker proteins (called genome tree) and with 16S rRNA genes (Fig. 1a, b, respectively) extracted from the genome. The results indicated that NAS-02 belonged to THSCG, and supported that THSCG branched out between the AOT clade and other archaeal clades, such as Crenarchaeota and $\mathrm{Ca}$. Aigarchaeota, as reported previously [14, 18]. NAS-02 clustered with the metagenome-assembled genomes of Beowulf BS1 and Dragon DS1 [18]. The closest $16 \mathrm{~S}$ rRNA gene sequence deposited in public databases to that of NAS-02 was the fosmid clone $1 \mathrm{~m} 19$ recovered from a Kamchatka thermoacidic spring, Russia [13] with a 99.3\% similarity. Based on the 16S rRNA gene phylogeny (Fig. 1b), NAS-02 was affiliated with Group I.1f/HTC2, a sub-clade of the THSCG [13, 18]. The closest 16S rRNA gene sequence of cultured species to that of NAS-02 was Hyperthermus butylicus (85.1\%), followed by Pyrolobus fumarii (84.8\%) and Ca. Nitrosocaldus yellowstonii $(83.3 \%)$.

Considering the phylogenetic positions of THSCG on the basis of the single copy marker proteins or the 16S rRNA gene, THSCG should represent a major clade different from AOT or class Nitrososphaera among the phylum Thaumarchaeota [50]. At present, NAS-02 is the sole cultivated member of THSCG, and we propose tentatively the name Conexivisphaera calidus to accommodate NAS-02 ( $=\mathrm{JCM}$ 31663). The genus will also represent the higher taxa (i.e.,

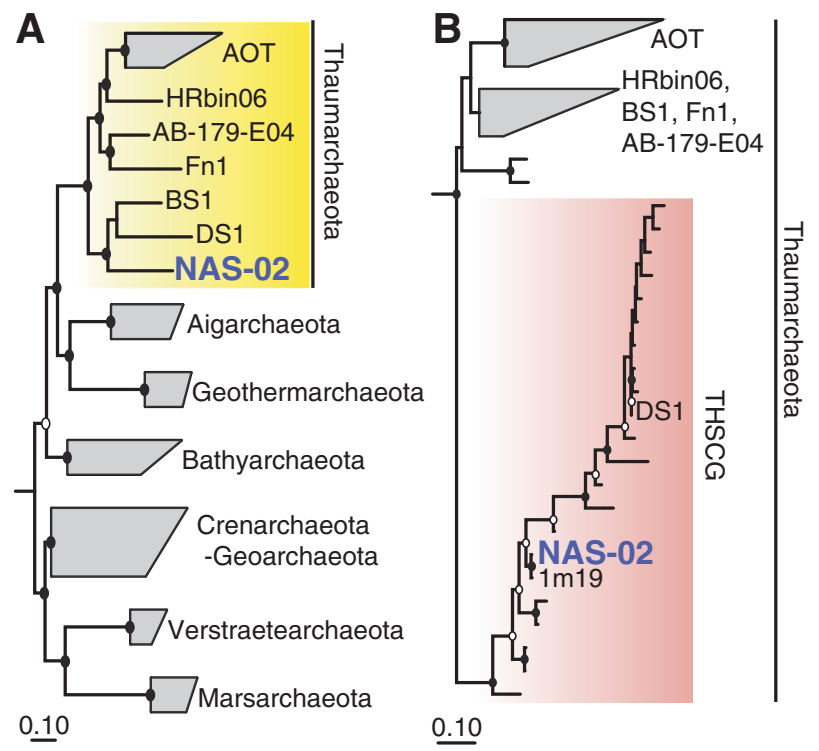

Fig. 1 Phylogenetic trees for NAS-02. The maximum-likelihood trees were constructed using RAxML for a concatenated single copy marker proteins (a total of 43 proteins) and for $\mathbf{b} 16 \mathrm{~S}$ rRNA genes. Bootstrap values are indicated as filled circles $(\geq 70 \%)$ and open circles $(\geq 50$ and $<70 \%$ ) at nodes. The scale bar represents 0.1 amino acid or nucleotide substitutions per sequence position. AOT, ammonia-oxidizing thaumarchaeota; THSCG, Terrestrial Hot Spring Crenarchaeotic Group
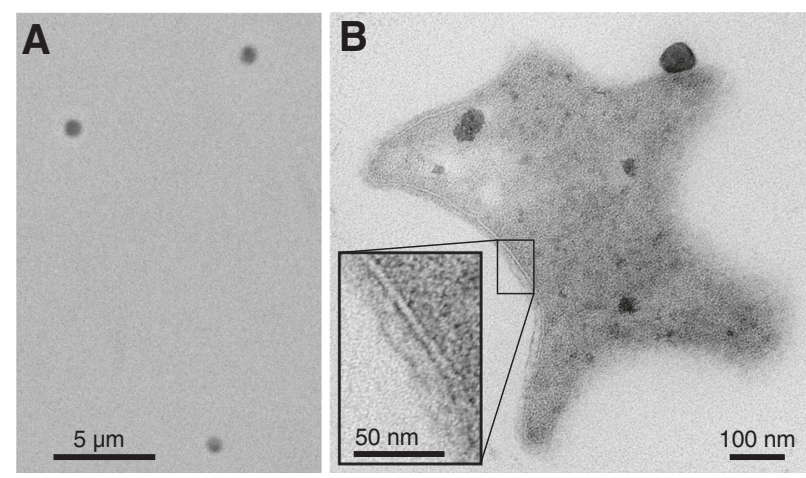

Fig. 2 Morphology of NAS-02 cells. a An image of phase-contrast microscopy. b An image of ultrathin-section transmission electron microscopy. S-layer and cell membrane were observed (enlarged image in the left bottom box). Particles showing high electron density were observed both inside and outside a cell

family, order and class) of the THSCG lineage within the phylum Thaumarchaeota.

Physiological characterization demonstrated that NAS-02 was a moderate thermophilic, weak acidophilic, ironand sulfur-reducing organoheterotrophic archaeon. Its physiology is summarized in Table 1. A growth curve of NAS02 cultivated with $\mathrm{S}_{2} \mathrm{O}_{3}{ }^{2-}$ as the electron acceptor at the optimal conditions $\left(65^{\circ} \mathrm{C}\right.$ and pH 5.0) is shown in Fig. S3. Its doubling time was $40 \mathrm{~h}$ at the exponential phase. The maximal cell density was $\sim 1.0 \times 10^{7}$ cells per mL. NAS-02 grew with sulfur species and $\mathrm{Fe}^{3+}$ as sole electron 
acceptors, but neither $\mathrm{NO}_{3}{ }^{-}$nor $\mathrm{O}_{2}$. Neither fermentative nor autotrophic growth was observed. It used proteinaceous substances (such as yeast extract and peptone) as the electron donors and carbon sources, but not the other organic carbon compounds tested (Table S2). Cells of NAS-02 were in irregular-coccoid shape, $\sim 0.5-0.8 \mu \mathrm{m}$ in diameter (Fig. 2). No motility was observed, and neither flagella-like nor pili-like structures was found. There were intracellular particles containing $\mathrm{S}, \mathrm{Fe}, \mathrm{As}, \mathrm{Se}$, and $\mathrm{P}$ in the cells growing with thiosulfate (Fig. S4). It is possible that sulfide is produced by thiosulfate reduction, and then precipitated as metal sulfide (or phosphate) within the cells. Such particles were also observed outside the cells (Fig. 2b, Fig. S4).

The genomic characteristics reflect the thermophily of NAS-02. The genome encodes a gene for reverse gyrase (NAS2_0035) that is a hallmark of (hyper)thermophiles [51]. This gene was also found in DS1, but not in other relatives (such as BS1, HRbin06, and AB-179-E04) from hot environments possibly due to incompleteness of these genomes. We estimated optimal growth temperature $\left(66^{\circ} \mathrm{C}\right)$ of NAS-02 based on its proteome-wide 2-mer amino acid composition [41], which was consistent with its actual growth temperature. In addition, the estimated optimal growth temperatures of its relatives were also consistent with the in situ temperatures of their habitats (Table 1). Thus, these results strongly support that the deep-branching lineages of Thaumarchaeota include thermophiles, as suggested previously [18].

Anabolic and catabolic metabolisms deduced from the annotatable CDSs (Fig. 3; Table S4) were consistent with the above physiological characterization. The NAS-02 genome encodes genes involved in peptide and fatty acid degradation and elemental sulfur/thiosulfate reduction. For the peptide degradation, genes encoding extracellular and intracellular peptidases, aminotransferases of branched amino acids and aspartate, glutamate dehydrogenase are likely involved. NAS-02 is also likely able to degrade fatty acids via beta oxidation pathway. The acetyl-CoA, oxaloacetate, and 2-oxoglutarate produced by degradation of peptides and fatty acids could be used for production of biomass. ATP and reduced cofactors, such as NADPH, reduced ferredoxin $\left(\mathrm{Fd}_{\mathrm{red}}\right)$, and quinol $(\mathrm{QH})$, could be produced via the Embden-Meyerhof-Parnas (EMP) pathway, pentose phosphate (PP) pathway, and tricarboxylic acid (TCA) cycle, of which complete gene sets were found in the genome. Acetate might be incorporated into acetyl-CoA via AMP-forming acetyl-CoA synthase (ACS) using ATP.

The NAS-02 genome encodes genes for NAD(P)H:sulfur oxidoreductase (Nsr), thiosulfate reductase / polysulfide reductase $(\mathrm{Phs} / \mathrm{Psr})$, and sulfur reductase (Sre), which are likely involved in the reduction of $\mathrm{S}^{0}$ and $\mathrm{S}_{2} \mathrm{O}_{3}{ }^{2-}$. The genome also encodes a gene for thiosulfate:quinone oxidoreductase (Tqo), which may be used for quinol oxidation and $\mathrm{S}_{2} \mathrm{O}_{3}{ }^{2-}$ reduction. In addition, we found genes for ferredoxin:NADP ${ }^{+}$oxidoreductase (FNR) and electron transfer flavoprotein (ETF):quinone oxidoreductase (Fix), which are likely involved in electron bifurcation. We found genes for $\mathrm{NAD}(\mathrm{P}) \mathrm{H}$ :quinone oxidoreductase (Nuo)-like complex. The Nuo-like complex contained homologs of subunits NuoA-D and $\mathrm{H}-\mathrm{N}$, but not NuoE, F, and G that are necessary to obtain electron from NAD(P)H. Such Nuolike complex without NuoE-G is thought to be a progenitor of Complex I as $\mathrm{Fd}_{\text {red }}$ :quinone oxidoreductase [52]. Indeed, a Nuo-like complex without NuoE-G in a methanogenic euryarchaeote Methanosaeta thermophila oxidizes $\mathrm{Fd}_{\mathrm{red}}$ [53]. Oxidation of the reduced cofactors on the cell membrane via the Sre, Phs/Psr, and/or Nuo-like complex could create proton motive force (PMF), and eventually, ATP could be generated by the ATPase using the PMF. The $\mathrm{H}^{+} / \mathrm{Na}^{+}$antiporter could be used for maintaining homeostasis of $\mathrm{pH}$ and salinity.

The NAS-02 genome encodes a total of 18 genes related to molybdoenzymes containing a molybdenum cofactor (MoCo) binding site (Fig. S5). Molybdoenzymes include a variety of oxidoreductases, which has been classified into three families, i.e., the xanthine oxidase (XO) family, dimethyl sulfoxide (DMSO) reductase family, and sulfite oxidase family [54]. The XO family includes carbon monoxide dehydrogenase (Cox) and aldehyde oxidoreductase (AOR), in addition to XO. The DMSO family includes formate dehydrogenase (Fdh), and Sre and Phs/Psr. The NAS-02 genome encodes 5 and 13 genes for the XO (Fig. S6) and DMSO families (Fig. S7A-F), respectively. Although genes of the XO family were also found in the genomes of the NAS-02 relatives (e.g., DS1 and BS1), those of DMSO families were rarely found in them possibly due to their incompleteness. Although these molybdoenzymes (except Sre and Phs/Psr) appeared to be involved in oxidation/reduction of carbon monoxide, organic carbon such as formate and aldehydes, and redox cofactors, their exact functions are still unclear because of no supporting physiological data. Further physiological and biochemical analyses are needed to reveal their functions.

The genome encodes cytochrome bd ubiquinol oxidase, Bcp-type thiol peroxidase, and superoxide dismutase, which might be involved in resistance to oxidative stress. Indeed, NAS-02 was tolerant to traces $\mathrm{O}_{2}$ (i.e., growth without a reducing regent, such as L-cysteine or sodium sulfide, into the media), but could not grow at $1 \%$ or higher $\mathrm{O}_{2}$ levels in the headspace.

Although NAS-02 grew on $\mathrm{Fe}^{3+}$, there were no homologs for known ferric iron reductase and multi-heme c-type cytochromes, which are involved in iron reduction in archaea and bacteria [55-57]. NAS-02 may have an unknown mechanism for $\mathrm{Fe}$ reduction, as reported for the other Fe-reducing archaea, such as Pyrobaculum 


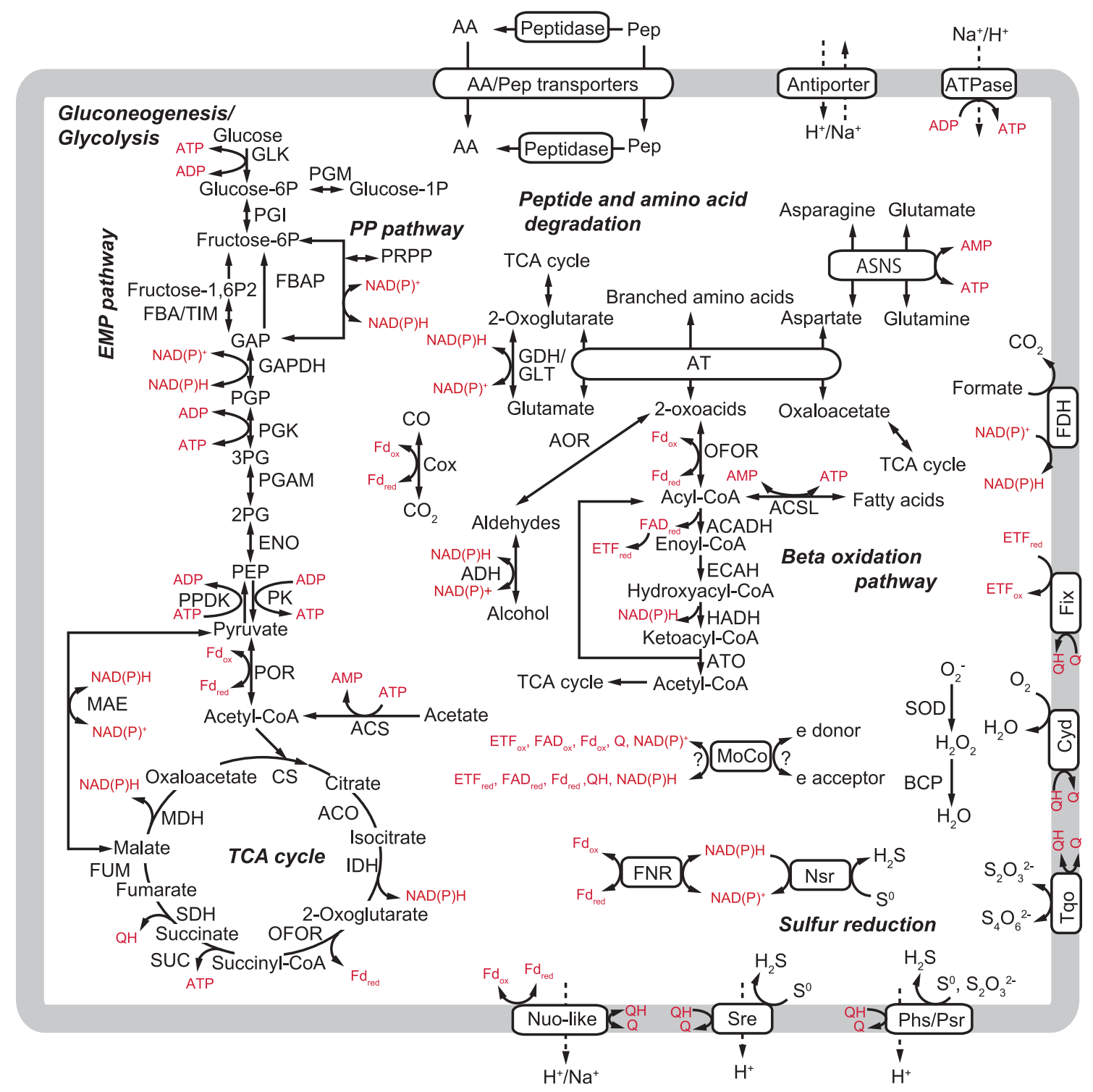

Fig. 3 Metabolisms deduced from annotatable CDSs. The list of the CDSs is shown in Table S4

spp., Pyrococcus spp. [58], and members in Ca. Marsarchaeota [6].

As reported in the nearly complete genomes of deeper lineages of Thaumarchaeota [18, 59, 60], the NAS-02 complete genome also lacked amo for ammonia monooxygenase, a key enzyme for ammonia oxidation [15]. This strongly supports that the deeper lineages of Thaumarchaeota do not represent ammonia oxidizers.

We focused on the evolutionary history of genes encoding Nsr, Sre, and Phs/Psr that are involved in reduction of sulfur compounds. The phylogenetic analysis (Fig. 4a) showed that Nsr of NAS-02 was located within a crenarchaeal clade of the class Thermoproteaceae. Although a $n s r$ was also found in DS1, it was phylogenetically distant from that of NAS-02 (Fig. 4a). The phylogenetic analysis of the catalytic subunit of Sre (Fig. 4b) showed that Sre of NAS-02 was located within a crenarchaeal clade of the class Sulfolobaceae. Neither phs/ psr nor sre has been found in the genomes of the deepbranching relatives (e.g., DS1 and BS1) of Thaumarchaeota so far. Based on the phylogenies (Fig. 4a, b), it seems that the lineage of NAS-02 has gained the genes of Nsr and Sre from members of Crenarchaeota via horizontal gene transfer (HGT). Indeed, the Nsr and Sre of NAS-02 were most closely related to those of Vulcanisaeta spp. of Thermoproteaceae and Acidianus spp. of Sulfolobaceae, of which 16S rRNA genes were detected in the sampling point by PCR clone library analysis (Fig. S8). In contrast, Phs/Psr of NAS-02, as well as those of a bacteria clade containing members (e.g., Sutterella spp. and Dakarella spp.) of the 
Fig. 4 Phylogenetic trees for CDSs involved in sulfur reduction. The maximumlikelihood trees were constructed using RAxML for a Nsr and b Sre and Phs/Psr. Taxonomic names at order and class levels for Crenarchaeota are shown. Stars indicate the presence of the same species in each tree. Bootstrap values are indicated as filled circles $(\geq 70 \%)$ and open circles $(\geq 50 \%$ and $<70 \%$ ) at nodes

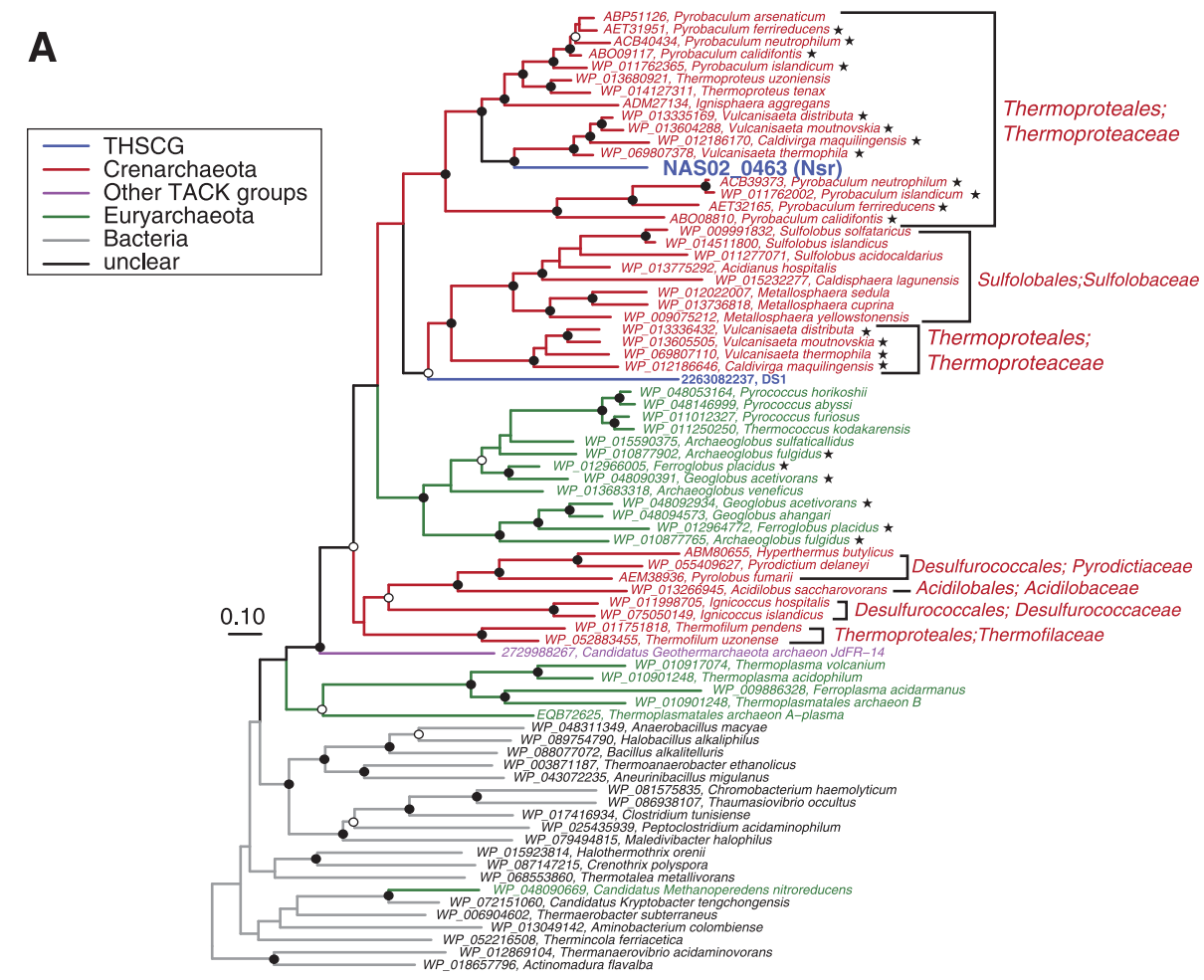

B

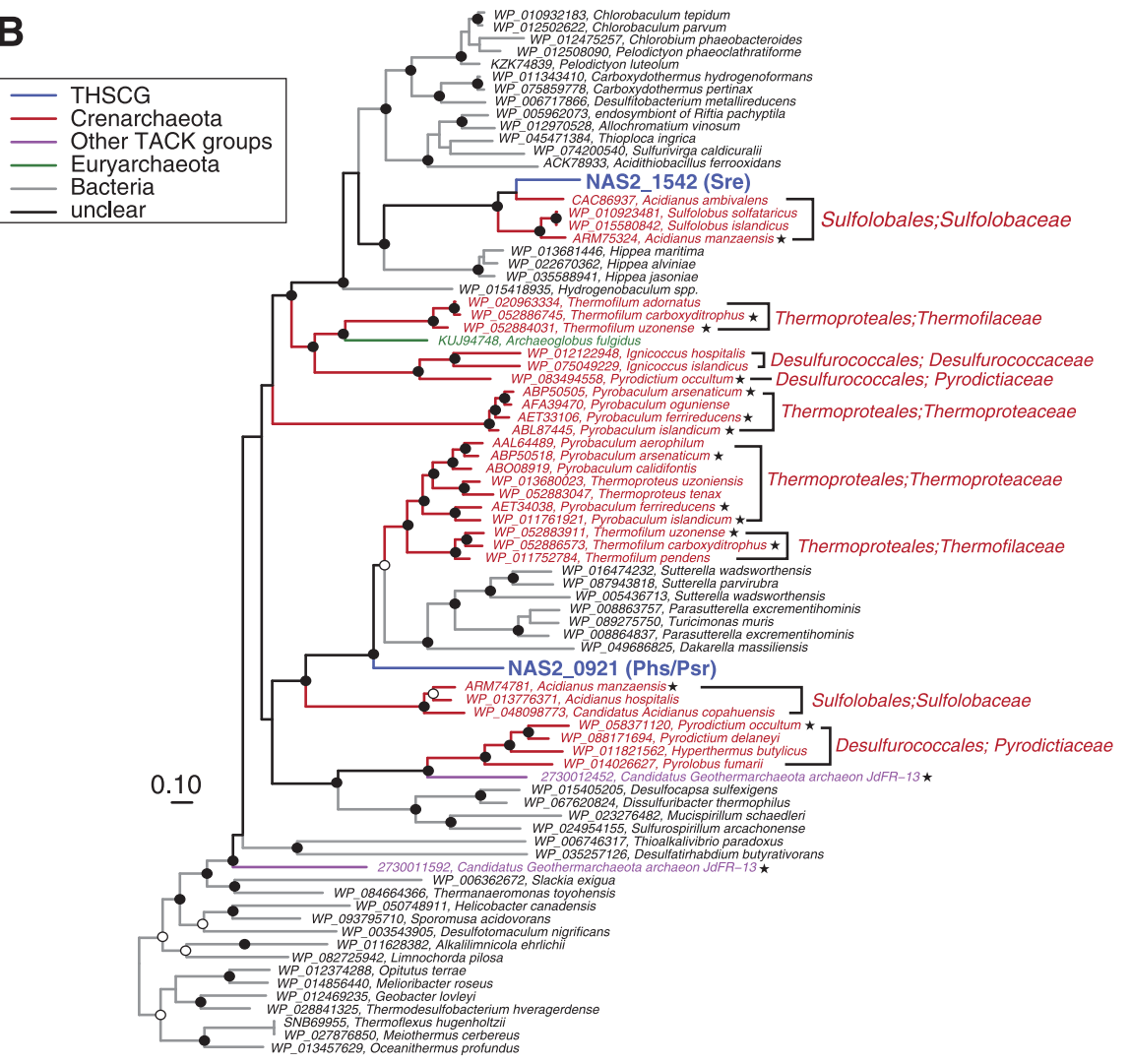

family Sutterellaceae of Betaproteobacteria, was located between crenarchaeal clades of Sulfolobales and Thermoproteales. If NAS- 02 had vertically gained the genes of Nsr,
Sre, and Phs/Psr from the common ancestor of THSCG and Crenarchaeota, the NAS-02 genes would have been located outside a large clade including Sulfolobales and 
Thermoproteales at least. It should be noted that the genes of Nsr, Sre, and Phs/Psr have likely experienced multiple events of gene duplication, gene loss, and HGT, as suggested by the phylogenetic trees (Fig. 4). Thus, the evolutionary history of the genes involved in sulfur reduction are complicated. Further determination and analysis of genome sequences of members in THSCG and relative clades are needed to reveal the detailed evolutionary history of the genes.

To assess geographical distribution of THSCG, we surveyed 16S rRNA gene sequences of THSCG in public databases. Although members of THSCG were hardly detected in the hot spring water obtained for this study, the 16S rRNA gene sequences of THSCG have been frequently detected in terrestrial acidic hot springs of several countries, such as Russia, China, Argentina and Japan, and also in marine hydrothermal fields, such as Okinawa Trough, Juan de Fuca Ridge, Mid Atlantic Ridge, and Mohn Ridge (Fig. S9). As consistent with the thermophily of NAS-02, the geographical distribution of THSCG appears to be restricted to thermal environments. Based on the NAS-02 physiology shown in the present study and the previous metagenomics insights [18], members in THSCG may play a role in degrading organic detritus as a "scavenger" and in geochemical cycling of sulfur and iron in such environments.

Acknowledgements We would like to thank the owner of the spa for permitting us to collect hot spring samples. We also would thank Dr. Kenshiro Oshima and Dr. Masahira Hattori (The University of Tokyo) for their help on genome sequencing. We would also like to thank anonymous reviewers for their helpful and insightful suggestions. This work was supported by RIKEN Special Postdoctoral Researchers Program, RIKEN Incentive Research Grant and Institute of Fermentation (IFO), Osaka, and partially by the Cabinet Office, Government of Japan, through the Next-generation Technology for Ocean Resources Exploration (called as Zipangu-in-the-ocean project) in the Cross-ministerial Strategic Innovation Promotion Program (SIP).

Author contributions S.K., T.I., and Mo.O. conceived this study. S.K., T.I., T.T., K.S., and Mo.O. supervised experiments and analyses. S.K., T.I., M.N., Ma.O., M.Y., and K.U. performed analyses. S.K., T.I., and Mo.O. wrote the paper with contributions from all authors.

\section{Compliance with ethical standards}

Conflict of interest The authors declare that they have no conflict of interest.

Publisher's note: Springer Nature remains neutral with regard to jurisdictional claims in published maps and institutional affiliations.

\section{References}

1. Rinke C, Schwientek P, Sczyrba A, Ivanova NN, Anderson IJ, Cheng JF, et al. Insights into the phylogeny and coding potential of microbial dark matter. Nature. 2013;499:431-7.
2. Castelle CJ, Wrighton KC, Thomas BC, Hug LA, Brown CT, Wilkins MJ, et al. Genomic expansion of domain archaea highlights roles for organisms from new phyla in anaerobic carbon cycling. Curr Biol. 2015;25:690-701.

3. Spang A, Saw JH, Jorgensen SL, Zaremba-Niedzwiedzka K, Martijn J, Lind AE, et al. Complex archaea that bridge the gap between prokaryotes and eukaryotes. Nature. 2015;521:173-9.

4. Seitz KW, Lazar CS, Hinrichs K-U, Teske AP, Baker BJ. Genomic reconstruction of a novel, deeply branched sediment archaeal phylum with pathways for acetogenesis and sulfur reduction. ISME J. 2016;10:1696-705.

5. Lazar CS, Baker BJ, Seitz KW, Teske AP. Genomic reconstruction of multiple lineages of uncultured benthic archaea suggests distinct biogeochemical roles and ecological niches. ISME J. 2017;11:1118-29.

6. Jay ZJ, Beam JP, Dlakic M, Rusch DB, Kozubal MA, Inskeep WP. Marsarchaeota are an aerobic archaeal lineage abundant in geothermal iron oxide microbial mats. Nat Microbiol. 2018;3:732-40.

7. Adam PS, Borrel G, Brochier-Armanet C, Gribaldo S. The growing tree of Archaea: new perspectives on their diversity, evolution and ecology. ISME J. 2017;11:2407-25.

8. Spang A, Caceres EF, Ettema TJG. Genomic exploration of the diversity, ecology, and evolution of the archaeal domain of life. Science. 2017; 357:eaaf3883.

9. Barns SM, Fundyga RE, Jeffries MW, Pace NR. Remarkable archaeal diversity detected in a Yellowstone National Park hot spring environment. Proc Natl Acad Sci USA. 1994;91:1609-13.

10. Kato S, Itoh T, Yamagishi A. Archaeal diversity in a terrestrial acidic spring field revealed by a novel PCR primer targeting archaeal 16S rRNA genes. FEMS Microbiol Lett. 2011;319:34-43.

11. Takai K, Sako Y. A molecular view of archaeal diversity in marine and terrestrial hot water environments. FEMS Microbiol Ecol. 1999;28:177-88.

12. Takai K, Horikoshi K. Genetic diversity of archaea in deep-sea hydrothermal vent environments. Genetics. 1999;152:1285-97.

13. Eme L, Reigstad LJ, Spang A, Lanzen A, Weinmaier T, Rattei T, et al. Metagenomics of Kamchatkan hot spring filaments reveal two new major (hyper)thermophilic lineages related to Thaumarchaeota. Res Microbiol. 2013;164:425-38.

14. Guy L, Ettema TJ. The archaeal 'TACK' superphylum and the origin of eukaryotes. Trends Microbiol. 2011;19:580-7.

15. Francis CA, Roberts KJ, Beman JM, Santoro AE, Oakley BB. Ubiquity and diversity of ammonia-oxidizing archaea in water columns and sediments of the ocean. Proc Natl Acad Sci USA. 2005;102:14683-8.

16. Wuchter C, Abbas B, Coolen MJ, Herfort L, van Bleijswijk J, Timmers $\mathrm{P}$, et al. Archaeal nitrification in the ocean. Proc Natl Acad Sci USA. 2006;103:12317-22.

17. Leininger S, Urich T, Schloter M, Schwark L, Qi J, Nicol GW, et al. Archaea predominate among ammonia-oxidizing prokaryotes in soils. Nature. 2006;442:806-9.

18. Beam JP, Jay ZJ, Kozubal MA, Inskeep WP. Niche specialization of novel Thaumarchaeota to oxic and hypoxic acidic geothermal springs of Yellowstone National Park. ISME J. 2014;8:938-51.

19. Itoh T, Onishi M, Kato S, Ino T, Sakamoto M, Kudo T, et al. Athalassotoga saccharophila gen. nov. sp. nov. isolated from an acidic terrestrial hot spring of Japan, and proposal of Mesoaciditogales ord. nov., Mesoaciditogaceae fam. nov. in the phylum Thermotogae. Int J Syst Evol Microbiol. 2015;66:1045-51.

20. Stookey LL. Ferrozine-a new spectrophotometric reagent for iron. Anal Chem. 1970;42:779-81.

21. Balch WE, Fox GE, Magrum LJ, Woese CR, Wolfe RS. Methanogens: reevaluation of a unique biological group. Microbiol Rev. 1979;43:260-96. 
22. Kato S, Kobayashi C, Kakegawa T, Yamagishi A. Microbial communities in iron-silica-rich microbial mats at deep-sea hydrothermal fields of the Southern Mariana Trough. Environ Microbiol. 2009;11:2094-111.

23. Itoh T, Kudo T, Oyaizu H, Seino A. Two new species in the genus Actinomadura: A. glomerata sp. nov., and A. longicatena sp. nov. Actinomycetologica. 1995;9:164-77.

24. Yamaguchi M, Namiki Y, Okada H, Uematsu K, Tame A, Maruyama T, et al. Improved preservation of fine structure of deep-sea microorganisms by freeze-substitution after glutaraldehyde fixation. J Electron Microsc (Tokyo). 2011;60:283-7.

25. Bankevich A, Nurk S, Antipov D, Gurevich AA, Dvorkin M, Kulikov AS, et al. SPAdes: a new genome assembly algorithm and its applications to single-cell sequencing. J Comput Biol. 2012;19:455-77.

26. Seemann T. Prokka: rapid prokaryotic genome annotation. Bioinformatics. 2014;30:2068-9.

27. Hyatt D, Chen GL, Locascio PF, Land ML, Larimer FW, Hauser LJ. Prodigal: prokaryotic gene recognition and translation initiation site identification. BMC Bioinformatics. 2010;11:119.

28. Nawrocki EP, Eddy SR. Infernal 1.1: 100-fold faster RNA homology searches. Bioinformatics. 2013;29:2933-5.

29. Lagesen K, Hallin P, Rodland EA, Staerfeldt HH, Rognes T, Ussery DW. RNAmmer: consistent and rapid annotation of ribosomal RNA genes. Nucleic Acids Res. 2007;35:3100-8.

30. Lowe TM, Chan PP. tRNAscan-SE on-line: integrating search and context for analysis of transfer RNA genes. Nucleic Acids Res. 2016;44:W54-57.

31. Overbeek R, Olson R, Pusch GD, Olsen GJ, Davis JJ, Disz T, et al. The seed and the rapid annotation of microbial genomes using subsystems technology (RAST). Nucleic Acids Res. 2014;42:D206-214.

32. Pelve EA, Martens-Habbena W, Stahl DA, Bernander R. Mapping of active replication origins in vivo in thaum- and euryarchaeal replicons. Mol Microbiol. 2013;90:538-50.

33. Altschul SF, Madden TL, Schaffer AA, Zhang J, Zhang Z, Miller W, et al. Gapped BLAST and PSI-BLAST: a new generation of protein database search programs. Nucleic Acids Res. 1997;25:3389-402.

34. Huson DH, Beier S, Flade I, Górska A, El-Hadidi M, Mitra S, et al. MEGAN community edition-interactive exploration and analysis of large-scale microbiome sequencing data. PLoS Comput Biol. 2016;12:e1004957.

35. Huson DH, Auch AF, Qi J, Schuster SC. MEGAN analysis of metagenomic data. Genome Res. 2007;17:377-86.

36. Huerta-Cepas J, Szklarczyk D, Forslund K, Cook H, Heller D, Walter MC, et al. eggNOG 4.5: a hierarchical orthology framework with improved functional annotations for eukaryotic, prokaryotic and viral sequences. Nucleic Acids Res. 2016;44: D286-293.

37. Ogata H, Goto S, Sato K, Fujibuchi W, Bono H, Kanehisa M. KEGG: Kyoto encyclopedia of genes and genomes. Nucleic Acids Res. 1999;27:29-34.

38. Zdobnov EM, Apweiler R. InterProScan-an integration platform for the signature-recognition methods in InterPro. Bioinformatics. 2001;17:847-8.

39. Yu NY, Wagner JR, Laird MR, Melli G, Rey S, Lo R, et al. PSORTb 3.0: improved protein subcellular localization prediction with refined localization subcategories and predictive capabilities for all prokaryotes. Bioinformatics. 2010;26:1608-15.

40. Rawlings ND, Barrett AJ, Finn R. Twenty years of the MEROPS database of proteolytic enzymes, their substrates and inhibitors. Nucleic Acids Res. 2016;44:D343-350.

41. Li G, Rabe KS, Nielsen J, Engqvist MKM Machine learning applied to predicting microorganism growth temperatures and enzyme catalytic optima. ACS Synth. Biol. 2019: https://doi.org/ 10.1021/acssynbio.9b00099

42. Quast C, Pruesse E, Yilmaz P, Gerken J, Schweer T, Yarza P, et al. The SILVA ribosomal RNA gene database project: improved data processing and web-based tools. Nucleic Acids Res. 2013;41:D590-596.

43. Pruesse E, Peplies J, Glockner FO. SINA: accurate highthroughput multiple sequence alignment of ribosomal RNA genes. Bioinformatics. 2012;28:1823-9.

44. Capella-Gutierrez S, Silla-Martinez JM, Gabaldon T. trimAl: a tool for automated alignment trimming in large-scale phylogenetic analyses. Bioinformatics. 2009;25:1972-3.

45. Stamatakis A. RAxML version 8: a tool for phylogenetic analysis and post-analysis of large phylogenies. Bioinformatics. 2014;30:1312-3.

46. Parks DH, Imelfort M, Skennerton CT, Hugenholtz P, Tyson GW. CheckM: assessing the quality of microbial genomes recovered from isolates, single cells, and metagenomes. Genome Res. 2015;25:1043-55.

47. Edgar RC. MUSCLE: multiple sequence alignment with high accuracy and high throughput. Nucleic Acids Res. 2004;32:1792-7.

48. Lagkouvardos I, Joseph D, Kapfhammer M, Giritli S, Horn M, Haller D, et al. IMNGS: a comprehensive open resource of processed 16S rRNA microbial profiles for ecology and diversity studies. Sci Rep. 2016;6:33721.

49. Caporaso JG, Kuczynski J, Stombaugh J, Bittinger K, Bushman FD, Costello EK, et al. QIIME allows analysis of high-throughput community sequencing data. Nat Methods. 2010;7:335-6.

50. Stieglmeier M, Klingl A, Alves RJ, Rittmann SK, Melcher M, Leisch N, et al. Nitrososphaera viennensis gen. nov., sp. nov., an aerobic and mesophilic, ammonia-oxidizing archaeon from soil and a member of the archaeal phylum Thaumarchaeota. Int J Syst Evol Microbiol. 2014;64:2738-52.

51. Forterre P. A hot story from comparative genomics: reverse gyrase is the only hyperthermophile-specific protein. Trends Genet. 2002;18:236-7.

52. Friedrich T, Scheide D. The respiratory complex I of bacteria, archaea and eukarya and its module common with membranebound multisubunit hydrogenases. FEBS Lett. 2000;479:1-5.

53. Welte C, Deppenmeier U. Membrane-bound electron transport in Methanosaeta thermophila. J Bacteriol. 2011;193:2868-70.

54. Leimkuhler S, Iobbi-Nivol C. Bacterial molybdoenzymes: Old enzymes for new purposes. FEMS Microbiol Rev. 2016;40:1-18.

55. Smith JA, Aklujkar M, Risso C, Leang C, Giloteaux L, Holmes DE. Mechanisms involved in $\mathrm{Fe}(\mathrm{III})$ respiration by the hyperthermophilic archaeon Ferroglobus placidus. Appl Environ Microbiol. 2015;81:2735-44.

56. Vadas A, Monbouquette HG, Johnson E, Schroder I. Identification and characterization of a novel ferric reductase from the hyperthermophilic archaeon Archaeoglobus fulgidus. J Biol Chem. 1999;274:36715-21.

57. Shi L, Dong H, Reguera G, Beyenal H, Lu A, Liu J, et al. Extracellular electron transfer mechanisms between microorganisms and minerals. Nat Rev Micro. 2016;14:651-62.

58. Lovley DR, Holmes DE, Nevin KP. Dissimilatory Fe(III) and Mn (IV) reduction. Adv Microb Physiol. 2004;49:219-86.

59. Lin X, Handley KM, Gilbert JA, Kostka JE. Metabolic potential of fatty acid oxidation and anaerobic respiration by abundant members of Thaumarchaeota and Thermoplasmata in deep anoxic peat. ISME J. 2015;9:2740-4.

60. Kato S, Sakai S, Hirai M, Tasumi E, Nishizawa M, Suzuki K, et al. Long-term cultivation and metagenomics reveal ecophysiology of previously uncultivated thermophiles involved in biogeochemical nitrogen cycle. Microbes Environ. 2018;33:107-10. 